\title{
Correction to: High resilience of the mycorrhizal community to prescribed seasonal burnings in eastern Mediterranean woodlands
}

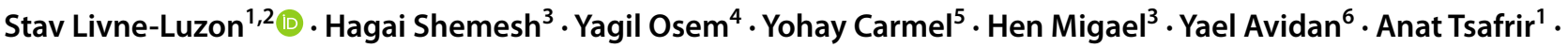 \\ Sydney I. Glassman ${ }^{7} \cdot$ Thomas D. Bruns $^{8}$. Ofer Ovadia ${ }^{1}$
}

Published online: 23 February 2021

๑) Springer-Verlag GmbH Germany, part of Springer Nature 2021

\section{Correction to: Mycorrhiza \\ https://doi.org/10.1007/s00572-020-01010-5}

The order of affiliations of the authors of the above article were rearranged and Ofer Ovadia one of the co-authors was added as co-corresponding author in the authorship group and affiliation section. The original article has been corrected.
The online version of the original article can be found https://doi. org/10.1007/s00572-020-01010-5.

\section{Stav Livne-Luzon}

stavl@weizmann.ac.il

$\bowtie$ Ofer Ovadia

oferovad@bgu.ac.il

1 Department of Life Sciences, Ben-Gurion University of the Negev, POB 653, Beer Sheva 84105, Israel

2 Department of Plant \& Environmental Sciences, Weizmann Institute of Science, Rehovot 76100, Israel

3 Department of Environmental Sciences, Tel-Hai College, Kiryat Shmona 1220800, Israel

4 Agricultural Research Organization, Volcani Center, Institute of Plant Sciences, Bet Dagan, Israel
Publisher's Note Springer Nature remains neutral with regard to jurisdictional claims in published maps and institutional affiliations.
5 Faculty of Civil and Environmental Engineering, TechnionIsrael Institute of Technology, Haifa, Israel

6 Mitrani Department of Desert Ecology, Swiss Institute for Dryland Environmental and Energy Research, The Jacob Blaustein Institutes for Desert Research, Ben-Gurion University of the Negev, Sede Boqer Campus 84990, Israel

7 Department of Microbiology and Plant Pathology, UC Riverside, Riverside, CA 92521, USA

8 Department of Plant and Microbial Biology, UC Berkeley, Berkeley, CA 94720-3102, USA 